\title{
Electrical Conductance Analysis of Solanum lycopersicum under Biotic Stress
}

\author{
1,2Teuma Mbezi M., ${ }^{4}$ Ambang Zachée, ${ }^{1}$ Ekobena Fouda H. and ${ }^{5}$ Kofané Timoleon C. \\ ${ }^{1}$ Laboratory of Biophysics, Department of Physics, Faculty of Sciences, University of Yaoundé I, \\ ,Yaoundé, Cameroon. \\ ${ }^{2}$ National Advanced School of Posts, Telecommunications and Information and Communication \\ Technologies. \\ ${ }^{4}$ Laboratory of Phytopathology, Department of biology and vegetal physiology Faculty of Sciences, \\ University of Yaoundé I, P.O. Box 812, Yaoundé, Cameroon \\ ${ }^{5}$ Laboratory of Mechanics, Department of Physics, Faculty of Sciences. \\ 3michelteuma@gmail.com; zachambang@yahoo.fr; \\ hekobena@gmail.com; tckofane@yahoo.com
}

\begin{abstract}
Our purpose is to provide different parameters of control from which one can identify a sick plant before the appearance of the first symptoms. We made a stochastic analysis and an analysis according to the theory of information, to deduce those characteristics parameters. It came out from our analysis that the DSP of health plant is above the DSP of the sick plant. Generally, the DSP of health and treated plant is above the DSP of sick and treated plant. However there is an overlapping between the DSP of sick and treated plant, and the health one for the whole value of the normalized reduced frequency. The average conductance of health plant is higher than the average conductance of sick plant. We also observed that, average conductance of health and treated plant is lower than the average conductance of sick and treated plant. The standard deviation of health plant is higher than the standard deviation of sick plant. We also observed that, standard deviation of health and treated plant is lower than the standard deviation of sick and treated plant. The electric conductance signal $\mathrm{G}(\omega, \mathrm{t})$ of Solanum lycopersicum leaf plant is not a statistics process in the broad sense $(\mathrm{SSL})$. Electric conductance $\mathrm{G}(\omega, \mathrm{t})$ of the plant is a non ergotic signal. The entropy of the sick plant is higher than the entropy of the health one. Those parameters can be used during the development of informatics application, and can be used in I.O.T. (internet of thing)
\end{abstract}

Key words: Statistics in the broad sense (SSL); ergotic; Solanum lycopersicum; spectral density of power (DSP); mildew; electric conductance; entropy.

\section{Introduction}

Now our days, organic matter is usually study by using electrical circuit [1-4]. Generally, one can identify the sick plants i.e. plants which had undergone a biotic or abiotic stress starting from the appearance of the symptoms on the plants; however the appearance of the symptoms supposes that the plant already underwent a certain number of damages inside their tissues; which could have an influence on the quality and quantity of resulting product produced from these plants [5]. Teuma et al measured the electrical resistance of tomato (Solanum lycopersicum) sheets infected by the mildew and untreated with the 
Teuma Mbezi M., Ambang Zachée, Ekobena Fouda H. and Kofané Timoleon C.; Electrical Conductance Analysis of Solanum Iycopersicum under Biotic Stress, Transactions on Machine Learning and Artificial Intelligence, Volume 8 No 2 April, (2020); pp: 1-10

ridomil $\mathrm{MC}$, of the infected and treated sheets; treated sheets but not infected and the pilot plants with an aim of using the biophysics methods to diagnose the physiological state of the plants subjected to the disease and the fungicidal treatent [4]. This study aims to firstly make a stochastic analysis [6]; secondly, an analysis according to the information theory, and to deduce from those analysis a characteristics parameters from which one can identify a sick plant to the health one before the apparition of visible symptoms.

\section{Materials and Methods}

Matlab software was used to analyze data.

\section{Methods of analysis}

\section{- Spectral density of power (DSP)}

The spectral density of power $\Upsilon_{G}(v)$ of $G(t)$ signal is expressed as:

$$
\Upsilon_{\mathrm{G}}(v)=\left|\widehat{\mathrm{G}}(v)^{2}\right|
$$

$$
\text { Where } \quad \widehat{\mathrm{G}}(v)=\sum_{n=1}^{N} G(n) e^{-j 2 \pi n v} \quad ; \quad G(n)=\frac{1}{R(n)}
$$

$\widehat{\mathrm{G}}(v)$ is the discrete time Fourier transformation of the electric conductance $\mathrm{G}(\mathrm{t})$ signal; $\mathrm{N}$ the number of $\mathrm{G}(\mathrm{t})$ sample ; $v$ is the normalized reduced frequency ; $v \in[0 ; 1[$.

The spectral density $\Upsilon_{G}(v)$ was evaluated firstly for the pilot plant (health plant); secondly for the health and treated plant with the ridomil MC; thirdly for the plant infected by the mildew and untreated with the ridomil MC; and fourthly, for the infected and treated plant. The resulting evaluations of the spectral density $\curlyvee \mathrm{G}(\mathrm{v})$ were each time plotted.

\subsection{Stochastic process analysis}

Since the measure value of $\mathrm{G}(\mathrm{t})$ is unpredictable during the 26 hours, we define a probabilized space $(\Omega, @, P)$, where $\Omega=\left\{\omega_{1}, \omega_{2}, \omega_{3}, \omega_{4}\right\}$ is the universe space, $@=p(\Omega)$ the entire parts of $\Omega$ and $P$ the probability of the plant to be health, or to be sick, or to be health and treated, or to be sick and treated.

We define the random variable $\omega$ which takes values:

$\omega=\omega_{1}=1$, when the plant is health,

$\omega=\omega_{2}=2$, when the plant is sick,

$\omega=\omega_{3}=3$, when the plant is health and treated,

$\omega=\omega_{4}=4$, when the plant is sick and treated.

We consider that $\mathrm{P}$ is equiprobable on $\Omega$; so we have:

$P=\frac{1}{4}=0.25$ i.e

$\mathrm{P}\left(\omega=\omega_{1}=1\right)=\mathrm{P}\left(\omega=\omega_{2}=2\right)=\mathrm{P}\left(\omega=\omega_{3}=3\right)=\mathrm{P}\left(\omega=\omega_{4}=4\right)=0.25$

We also define $E$ as the whole possible states of the process, and $E_{1}, E_{2}, E_{3}, E_{4}$ as: 
$E=\bigcup_{i=1}^{4} E_{i}$,

where $E_{1}, E_{2}, E_{3}$ and $E_{4}$ are respectively the whole possible state of the process to be: health, sick, health and treated, sick and treated.

T represents the whole discrete time.

We now define the electric conductance process $G(\omega, t)$ as:

$$
\begin{gathered}
\mathrm{G}: \Omega \times \mathrm{T} \rightarrow \mathrm{E} \\
(\omega, t) \rightarrow \mathrm{G}(\omega, \mathrm{t})
\end{gathered}
$$

Where $\mathrm{G}(\omega, \mathrm{t})=G_{t}(\omega)=\left\{\begin{array}{c}\text { E1 if } \omega=\omega 1, \forall t \in T \\ E 2 \text { if } \omega=\omega 2, \quad \forall t \in T\end{array}\right.$

$$
\mathrm{G}(\omega, \mathrm{t})=G_{t}(\omega)=\left\{\begin{array}{c}
\mathrm{E} 3 \text { if } \omega=\omega 3, \forall t \in T \\
E 4 \text { if } \omega=\omega 4, \quad \forall t \in T
\end{array}\right.
$$

$T=\{1,2,3,4,5,6,7,8,9,10 \ldots, 16\} ; g_{i, j}$ is the $j^{\text {em }}$ element of $\mathrm{Ei}$;

$i \in\{1,2,3,4\} ; j \in\{1,2,3,4,5,6,7,8,9,10 \ldots, 16\}$;

Let us suppose: $\hat{g}_{m, n}$ the $m \times n$ matrix of extra- cellular space conductance, and $g_{i, j}$ one of its element; with $m=4, n=26$. We then have:

$$
\hat{\mathrm{g}}_{m, m}=\left(g_{i, j}\right) 1 \leq i \leq m \leq j \leq n \quad \text { with }\left(g_{i, j}\right)=G\left(\omega=\omega_{i}, t=j\right)
$$

\section{Statistical properties:}

$$
\begin{aligned}
& \text { Average : } \quad \boldsymbol{M}_{\boldsymbol{R}}(\boldsymbol{t})=\sum_{i=1}^{4} G_{t}\left(\omega_{i}\right) \times P\left(\omega=\omega_{i}\right) \text { i.e } \\
& \boldsymbol{M}_{\boldsymbol{R}}(\boldsymbol{t}=\boldsymbol{j})=\sum_{\boldsymbol{i}=\mathbf{1}}^{\mathbf{4}} g_{i, j} \times P\left(\omega=\omega_{i}\right) \text { i.e } \\
& \boldsymbol{M}_{\boldsymbol{R}}(\boldsymbol{t}=\boldsymbol{j})=\frac{\mathbf{1}}{\mathbf{4}}\left(g_{1, j}+g_{2, j}+g_{3, j}+g_{4, j}\right)
\end{aligned}
$$

- Autocorrelation function:

$$
\tau_{G}(t=i, t=i+k)=\sum_{i=1}^{4} \sum_{l=1}^{4} g_{i, j} \times g_{l+k, j} \times P\left(\omega=\omega_{i}, \omega=\omega_{l}\right)
$$

Where:

$$
\begin{aligned}
& P\left(\omega=\omega_{1}, \omega=\omega_{2}\right)=P\left(\omega=\omega_{1}, \omega=\omega_{4}\right)=P\left(\omega=\omega_{2}, \omega=\omega_{3}\right)=0, \\
& P\left(\omega=\omega_{2}, \omega=\omega_{1}\right)=P\left(\omega=\omega_{4}, \omega=\omega_{1}\right)=P\left(\omega=\omega_{3}, \omega=\omega_{2}\right)=0 \\
& P\left(\omega=\omega_{4}, \omega=\omega_{3}\right)=P\left(\omega=\omega_{3}, \omega=\omega_{4}\right)=0
\end{aligned}
$$

because the plant can't be in health and sick at the same time, and

$$
\begin{aligned}
& P\left(\omega=\omega_{1}, \omega=\omega_{3}\right)=P\left(\omega=\omega_{2}, \omega=\omega_{4}\right)=\frac{2}{16}, \\
& P\left(\omega=\omega_{3}, \omega=\omega_{1}\right)=P\left(\omega=\omega_{4}, \omega=\omega_{2}\right)=\frac{2}{16}
\end{aligned}
$$

The conjoint probability of plant to be respectively healthy and treated, sick and treated

$$
\begin{aligned}
& P\left(\omega=\omega_{1}, \omega=\omega_{1}\right)=P\left(\omega=\omega_{2}, \omega=\omega_{2}\right)=\frac{2}{16}, \\
& P\left(\omega=\omega_{3}, \omega=\omega_{3}\right)=P\left(\omega=\omega_{2}, \omega=\omega_{3}\right)=\frac{2}{16} ;
\end{aligned}
$$


$\mathrm{k} \in N ; \mathrm{i}+\mathrm{k} \leq 4$, and $\mathrm{l}+\mathrm{k} \leq 4$.

Considering the above assumption, we then have:

$$
\begin{aligned}
& \tau_{G}(t=i, t=i+k)=\frac{1}{8}\left(g_{1, j} \times g_{1+k, j}+g_{2, j} \times g_{2+k, j}+g_{3, j} \times g_{3+k, j}+g_{4, j} \times g_{4+k, j}+g_{1, j} \times\right. \\
& \left.g_{3+k, j}+g_{3, j} \times g_{1+k, j}+g_{2, j} \times g_{4+k, j}+g_{4, j} \times g_{2+k, j}\right)
\end{aligned}
$$

\section{Temporal properties:}

- Temporal average:

$$
\mu_{G}\left(\omega=\omega_{i}\right)=\frac{1}{N}\left(\sum_{j=1}^{16} g_{i, j}\right)
$$

We deduced the average vector $\mathbf{M}\left(\mu_{G}(\omega=1), \mu_{G}(\omega=2), \mu_{G}(\omega=3), \mu_{G}(\omega=4)\right)$ and the standard deviation vector $\sigma(\sigma(\omega=1), \sigma(\omega=2), \sigma(\omega=3), \sigma(\omega=4))$

\section{- Temporal autocorrelation function:}

$$
\mu_{G G}\left(\omega=\omega_{i}\right)=\frac{1}{N}\left(\sum_{j=1}^{16} g_{i, j} \times g_{i, j+k}\right)
$$

$\mathrm{k} \in N ; \mathrm{j}+\mathrm{k} \leq 26$

\subsection{Analysis according to information theory evaluation of the entropy}

The average information quantity is evaluated by the entropy, expressed as:

$$
H(X)=-\sum_{i=1}^{N} P_{i} \log \left(P_{i}\right)
$$

Where $P_{i}$ is the probability of obtaining alphabet $\mathrm{x}_{\mathrm{i}}$.

The entropy was evaluated for two states of tomato plant.

\section{Results and Discussion}

We observe an oscillation of the electric conductance $\mathrm{G}(\mathrm{t})$ whether the plant is health, sick, health and treated, or sick and treated (Figure1). The picks of conductance of sick and treated plant are higher than the conductance picks of sick and untreated plant. The conductance picks of sick and untreated plant are generally up to the electric conductance of the health plant.

\section{- Spectral density of power of the electric conductance.}

By using equations 1, 2, 3, we have:

$$
\Upsilon_{G}(v)=\sqrt{\left(A^{2}(v)+B^{2}(v)\right)^{2}+4\left(A^{2}(v) B^{2}(v)\right)}
$$

Concerning the health plant, we have: 


$$
\begin{aligned}
& A(v)=\sum_{j=1}^{16} g_{1, j} \cos 2 \pi v j \\
& B(v)=\sum_{j=1}^{16} g_{1, j} \sin 2 \pi v j
\end{aligned}
$$

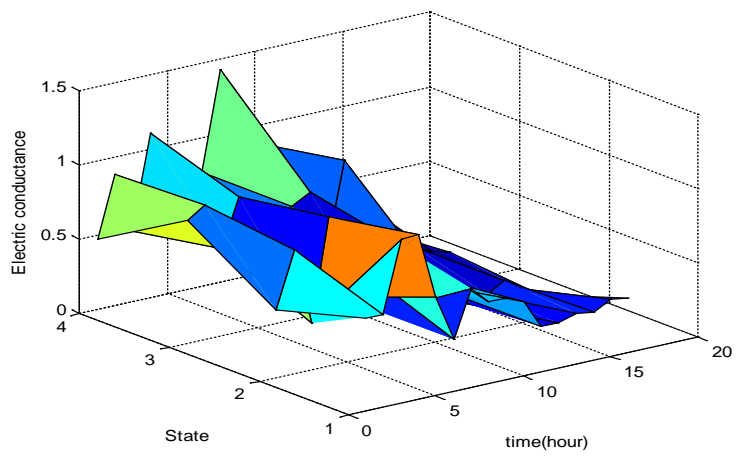

Figure 1: Behavior of electric conductance of plan under biotic stress. We observe an oscillation of the electric conductance $G(t)$ whether the plant is health, sick, health and treated, or sick and treated. The picks of conductance of sick and treated plant are higher than the conductance picks of sick and untreated plant. The conductance picks of sick and untreated plant are generally up to the electric conductance of the health, and sick plant. The conductance maxima of sick plant are also generally up the health one.

Concerning the sick plant, we have:

$$
\begin{aligned}
& A(v)=\sum_{j=1}^{16} g_{2, j} \cos 2 \pi v j \\
& B(v)=\sum_{j=1}^{16} g_{2, j} \sin 2 \pi v j
\end{aligned}
$$

Concerning the health and treated plant, we have:

$$
\begin{aligned}
& A(v)=\sum_{j=1}^{16} g_{3, j} \cos 2 \pi v j \\
& B(v)=\sum_{j=1}^{16} g_{3, j} \sin 2 \pi v j
\end{aligned}
$$

Concerning sick and treated plant, we have:

$$
\begin{aligned}
& A(v)=\sum_{j=1}^{16} g_{4, j} \cos 2 \pi v j \\
& B(v)=\sum_{j=1}^{16} g_{4, j} \sin 2 \pi v j
\end{aligned}
$$


Teuma Mbezi M., Ambang Zachée, Ekobena Fouda H. and Kofané Timoleon C.; Electrical Conductance Analysis of Solanum Iycopersicum under Biotic Stress, Transactions on Machine Learning and Artificial Intelligence, Volume 8 No 2 April, (2020); pp: 1-10

We can observe on Figure 2 that the DSP of health plant (green curve) is above the DSP of the sick plant (red curve). We can also observe that generally, the DSP of health and treated plant (yellow curve) is above the DSP of sick and treated plant (blue curve). However there is an overlapping between the DSP of sick and treated plant, and the health one for the whole value of the normalized reduced frequency.

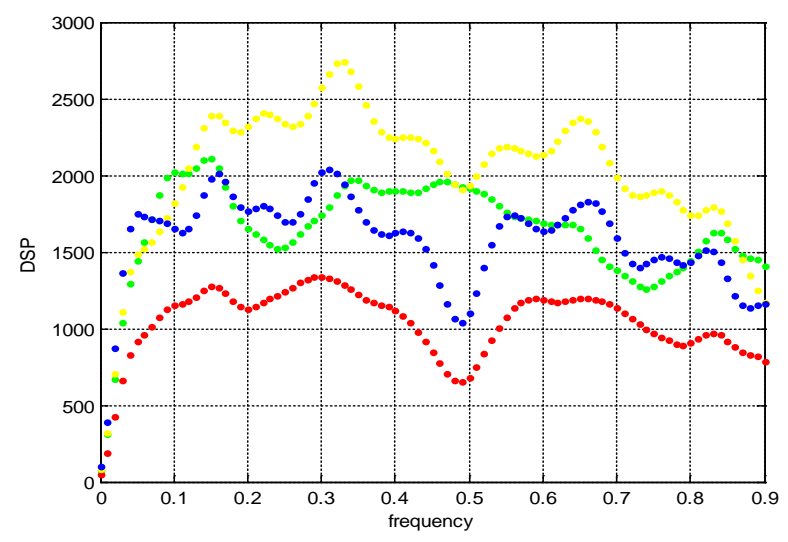

Figure 2. Spectral density of power of the electric conductance process $\mathrm{G}(\omega, \mathrm{t})$ of Solanum lycopersicum leaf plant. We can observe that the DSP of health plant (green curve) is above the DSP of the sick plant (red curve). We can also observe that generally, the DSP of health and treated plant (yellow curve) is above the DSP of sick and treated plant (blue curve). However there is an overlapping between the DSP of sick and treated plant, and the health one for the whole value of the normalized reduced frequency.

\section{Statistical properties:}

\section{- Statistical average}

In Figure3, the curve reveals that the electric conductance process $G(\omega, t)$ is non-statistics in the broad sense (non SSL) ; due to the fact that the statistical average of the $G(\omega, t)$ process Is not constant during the time of evolution. The statistical average has an oscillatory behavior which decreases according to time.

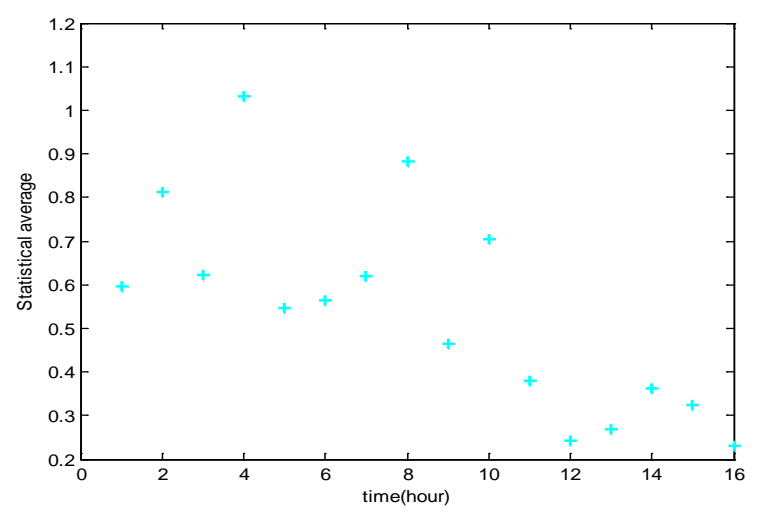

Figure 3. Statistical average of electric conductance signal $G(\omega, t)$ according to time of Solanum lycopersicum leaf plant. The curve reveals that the electric conductance process $\mathrm{G}(\omega, \mathrm{t})$ is non-statistics in the broad sense (non SSL) ; due to the fact that the statistical average of the $G(\omega, t)$ process Is not constant during the time evolution. The statistical average has an oscillatory behavior which decreases according to time. 


\section{- $\quad$ Autocorrelation function}

The autocorrelation decreases when we pass from the state 1 to the state 4 . The signal $G(\omega, t)$ of the health plant (state 1 ) is more correlate to the signal $G(\omega, t)$ of the sick plant (state 2 ) than the signal $G(\omega, t)$ of the sick and treated (state 3), and the health and treated (state 4) plant. The signal $G(\omega, t)$ doesn't depend only to displacement parameter $\mathrm{k}$ when passing from the health plant (state 1 ) to the health and treated plant (state 4), but also on time; it is not stationary as it is shown in the Figure 4 . This implies that the electric conductance signal $\mathrm{G}(\omega, \mathrm{t})$ of Solanum lycopersicum leaf plant is not a statistics process in the broad sense (SSL).

\section{Temporal properties:}

\section{- Temporal average and standard deviation}

The obtained average vector of electric conductance signal $G(\omega, t)$ is:

$\mathbf{M}(0.423 \mu S, 0.342 \mu S, 0.453 \mu S, 0.492 \mu S)$

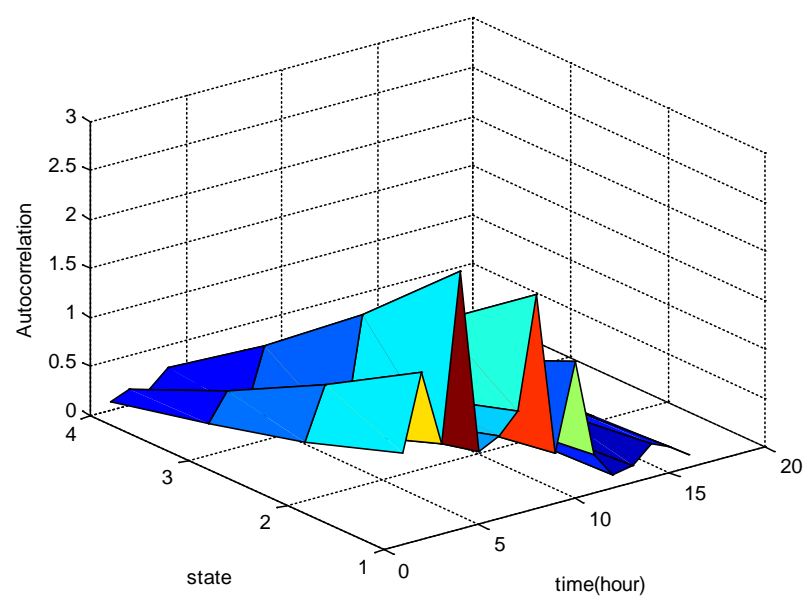

Figure 4. Statistical autocorrelation of electric conductance signal $G(\omega, t)$ according to time and the state of Solanum lycopersicum leaf plant. The autocorrelation decreases when we pass from the state 1 to the state 4 . The signal $G(\omega, t)$ of the health plant (state 1 ) is more correlate to the signal $G(\omega, t)$ of the sick plant (state 2 ) than the signal $G(\omega, t)$ of the sick and treated (state 3$)$, and the health and treated (state 4) plant. The signal $G(\omega, t)$ doesn't depend only to displacement parameter $k$ when passing from the health plant (state 1) to the health and treated plant (state 4), but also on time; it is not stationary as it is shown in the figure. This implies that the electric conductance signal $\mathrm{G}(\omega, \mathrm{t})$ of Solanum lycopersicum leaf plant is not a statistics process in the broad sense (SSL).

The average conductance of health plant is higher than the average conductance of sick plant. We also observed that, average conductance of health and treated plant is lower than the average conductance of sick and treated plant.

The standard deviation vector of the process is:

$\sigma(0.255,0.239,0.276,0.337)$ 
Teuma Mbezi M., Ambang Zachée, Ekobena Fouda H. and Kofané Timoleon C.; Electrical Conductance Analysis of Solanum Iycopersicum under Biotic Stress, Transactions on Machine Learning and Artificial Intelligence, Volume 8 No 2 April, (2020); pp: 1-10

The standard deviation of health plant is higher than the standard deviation of sick plant. We also observed that, standard deviation of health and treated plant is lower than the standard deviation of sick and treated plant.

\section{- Temporal autocorrelation function}

Autocorrelation has an oscillatory behavior according to time. Autocorrelation is higher for the health plant (state 1) than the other states. The lower autocorrelation is observed for the sick plant (state 2). Autocorrelation depends at the same time of the parameter of time evolution and the state of the plant; this implies that electric conductance $G(\omega, t)$ of the plant is a non ergotic signal (Figure5).

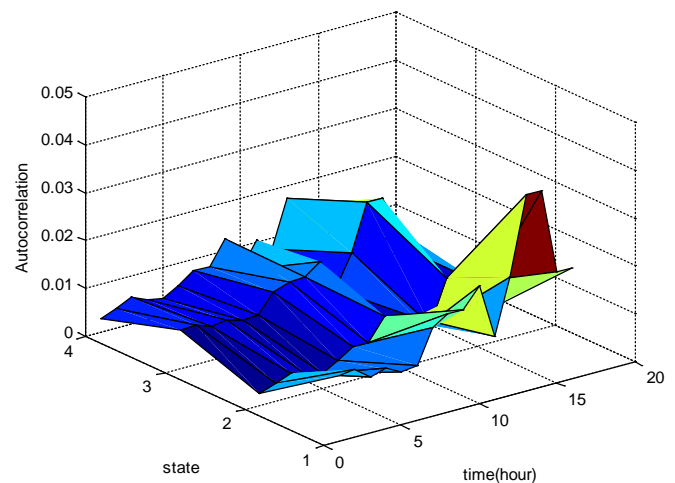

Figure 5. Temporal autocorrelation function according to time and the state of Solanum lycopersicum leaf plant. Autocorrelation has an oscillatory behavior according to time. Autocorrelation is higher for the health plant (state 1) than the other states. The lower autocorrelation is observed for the sick plant (state 2). Autocorrelation depends at the same time of the parameter of time evolution and the state of the plant; this implies that electric conductance $\mathrm{G}(\omega, \mathrm{t})$ of the plant is a non ergotic signal.

\section{evaluation of the entropy}

- Entropy of the health plant

\begin{tabular}{rl|l|l|l|l|l|l|l|l|l|l|l|}
\hline Alphabet $\mathrm{xi}$ & 0.625 & 1.111 & 0.666 & 0.357 & 0.555 & 0.5 & 0.312 & 0.303 & 0.344 & 0.322 & 0.384 & 0.37 \\
\hline $\mathrm{P}(\mathrm{X}=\mathrm{xi})$ & $2 / 16$ & $2 / 16$ & $2 / 16$ & $1 / 16$ & $2 / 16$ & $1 / 16$ & $1 / 16$ & $1 / 16$ & $1 / 16$ & $1 / 16$ & $1 / 16$ & $1 / 16$ \\
\hline & $H(X)=-\sum_{i=1}^{N=12} P_{i} \log \left(P_{i}\right)=1.520$ sh
\end{tabular}

- Entropy of the sick plant

\begin{tabular}{|l|l|l|l|l|l|l|l|l|l|l|l|l|l|}
\hline Alphabet xi & 0.454 & 0.833 & 0.312 & 1.0 & 0.434 & 0.588 & 0.277 & 0.714 & 0.303 & 0.204 & 0.243 & 0.227 & 0.161 \\
\hline $\mathrm{P}(\mathrm{X}=\mathrm{xi})$ & $1 / 16$ & $1 / 16$ & $2 / 16$ & $1 / 16$ & $3 / 16$ & $1 / 16$ & $1 / 16$ & $1 / 16$ & $1 / 16$ & $1 / 16$ & $1 / 16$ & $1 / 16$ & $1 / 16$ \\
\hline
\end{tabular}

$$
H(X)=-\sum_{i=1}^{N=13} P_{i} \log \left(P_{i}\right)=3.577 \mathrm{sh}
$$

Knowing that the passive electric characteristics reflect the degree of viability of life cells [7], one can say that electric conductance reveals the level of viability and vitality of a life cells. The fact that the means conductance of health plant $(0.423 \mu S)$ is higher than the means conductance of the sick one $(0.342 \mu S)$ 
revealed that the infection has decreased the vitality of the plant. One can also say that the infection of the plant by phytophthora (pathogenic agent of the mildew) perturbs the electric fluctuation of plant; what results in the lower standard deviation of the sick plant (0.239) than the health one (0.255). However the treatment of the sick plant with the ridomil MC fungicide increases the vitality and viability of plant which is revealed through the higher values of conductance means $(0.492 \mu S)$ and standard deviation (0.337). The ridomil MC fungicide also increases the vitality and viability of the health plant due to the higher values of conductance means $(0.453 \mu S)$ and standard deviation $(0.276)$ of the health and treated plant than conductance means and standard deviation of the health and no treated plant with ridomil.

When the plant was infected, a self-defense mechanism is started by the plant, which results in the higher conductance picks of the sick plant than the health plant (Figure 1). However the highest conductance picks observed in sick and treated plant can be due to the combine action of the self-defense mechanism and the addition of ions in the plant which came from ridomil.

The fact that the DSP of health plant is above the DSP of the sick plant reveals that, the infection of the plant decreases its electrical energy. The treatment of health and sick plant which ridomil increases their DSP i.e. their electrical energy. However, ridomil increases the energy of sick plant to the level of health plant. That is why we can observe there an overlapping between the DSP of sick and treated plant and the health one (Figure 2).

The fact that the statistical average of the $G(\omega, t)$ process is not constant during the time evolution may be explained by life nature of plant, i.e. the plant is not an inert matter. The physiological process of our four groups of plants is not uniform during the time evolution (Figure3).

The statistical autocorrelation, when there exists, is accentuated between the health plant and the sick one (Figure4). It reveals the level of reciprocal dependence between the conductance of health plant and the conductance of the sick one. The temporal autocorrelation is higher for the health plant than the sick one (Figure5). The weak correlation observed in the electric conductance may be due to the perturbation of phytophthora (pathogenic agent of the mildew).

Knowing that, the entropy reveals the information quantity, one can say that the entropy of the health plant (1.520) reveals the information quantity directly linked to the physiological activity of the health plant. However, when the plant is infected, the plant will activate a new physiological activity for it selfdefense; this will result to the additional information quantity (3.577).

\section{Conclusion}

The main concerned of our study was to provide different control parameters from which one can identify a sick plant before the appearance of the first symptoms. The tomato plants were set out in 4 groups. The first group was made up of plants into good health, the second group of the sick plants, the third group of plants into good health but treated by the ridomil, and the forth group was made up of sick plants which were treated by the ridomil MC fungicide. It came out from our analysis that the conductance picks of sick plant are generally up to the electric conductance of the health one. The picks of conductance of sick and treated plant are higher than the conductance picks of sick and untreated plant. The DSP of health plant is above the DSP of the sick plant. Generally, the DSP of health and treated plant is above the DSP of sick and treated plant. However there is an overlapping between the DSP of sick and treated plant, and the health one for the whole value of the normalized reduced frequency. The average conductance 
Teuma Mbezi M., Ambang Zachée, Ekobena Fouda H. and Kofané Timoleon C.; Electrical Conductance Analysis of Solanum Iycopersicum under Biotic Stress, Transactions on Machine Learning and Artificial Intelligence, Volume 8 No 2 April, (2020); pp: 1-10

of health plant is higher than the average conductance of sick plant. We also observed that, average conductance of health and treated plant is lower than the average conductance of sick and treated plant. The standard deviation of health plant is higher than the standard deviation of sick plant. We also observed that, standard deviation of health and treated plant is lower than the standard deviation of sick and treated plant. The electric conductance signal $\mathrm{G}(\omega, \mathrm{t})$ of Solanum lycopersicum leaf plant is not a statistics process in the broad sense (SSL). Electric conductance $G(\omega, t)$ of the plant is a non ergotic signal. The entropy of the sick plant is higher than the entropy of the health one.

\section{ACKNOWLEDGEMENTS}

We would like to express a special thanks to Pr Ben Boli Germain, the head of Atomic Molecular and Nuclear research group of U.Y.1; the head of Nuclear Technology Section of Cameroon Pr Saidou and the german teacher Nkolo Laure Martine.

\section{REFERENCES}

[1] Teuma Mbezi M, Ekobena Fouda H.P., Tabi Conrad B. and T.C. Kofane. Estimated Photosynthetic Activity from Its Electrical Impedance Spectroscopy. A.S.R.J.E.T.S. 2015, 13(1), pp.178-193.

[2] Teuma Mbezi M, Ekobena Fouda H.P., Tabi Conrad B. and T.C. Kofane. Estimated Photosynthesic Activity From its Passive Electrical Properties. International Journal of current. 2015, (7)10, pp. 21180-21185.

[3] Teuma Mbezi M, Ekobena Fouda H.P., Tabi Conrad B. and T.C. Kofane. Behavior of electrical properties of synthetic chorophyll pigment solution by using the T.E-model. Indian Journal of Science and Technology. 2017, 10(38).

[4] Teuma Mbezi M, Ekobena Fouda H.P., Ambang Zachée, Tabi Conrad B. and T.C. Kofane. study of the passive electrical properties of tomato tissues after infection and treatment by fongicide. Indian Journal of Science and Technology. 2017, 10(26).

[5] Philippe Lepoivre. Phytopathologie ; Bases moléculaires et biologiques des pathosystèmes et fondements des stratégies de lutte.2003; Editions De Boeck Université.

[6] Teuma Mbezi M, Ambang Zachée, Ekobena Fouda H.P., Tabi Conrad B. and T.C. Kofane. Stochastic Electrical Behavior of Splina Liquid Chlorophyll Drink Indian Journal of Science and Technology, Vol 11(12), DOI: 10.17485/ijst/2018/v11i12/120086, March2018

[7] Gounar II, Panishkmine LA, Tihonov FP. Activité bioélectrique du tournesol et de la tomate sur quelques types de pathogénéité. Jounal de l'académie agricole de Moscou. 1974; 5:3-8. 